\title{
Da Pluto alla Fata Plutina \\ (Italia liberata dai Goti)
}

Ludovica Radif

(Palacký University in Olomouc)

From Plutus to Plutina ("Italia liberata dai Goti")

\begin{abstract}
In attempting to investigate factors which may account for Aristophanes' revival in Renaissance times, the focus of this paper will be on Trissino's production, his reusing of passages and comic expressions from Plutus, in particular in the Italia liberata dai Goti. The primary goal of this study is to demonstrate that we are not talking about generic archaizing surfacing film, but concrete match to entire tracks, where Italian poet translates some Greek lines adjusting both traits and style to new different contest as well as audience (in greater detail, instead of the god and his distribution of wealth we see, here, a fairy and her spreading love...). This Trissino's personal way of approaching Aristophanes and entering lines from Plutus in the middle of his epic adventures proves that he quoted comedy regardless of topic but mostly like precious flourish.
\end{abstract}

\section{Keywords}

Trissino; Aristophanes in XVI century; Plutus in Renaissance time; Italia liberata dai Goti 
In parallelo all'indagine, di prossima uscita, compiuta sulla commedia Simillimi di Trissino, vorrei qui evidenziare il ritorno di un passo del Pluto di Aristofane all'interno del poema epico l'Italia liberata dai Goti.

In quest'opera (1547), dedicata a Carlo V, il Trissino, come noto, intendeva riprodurre la formula tradizionale del poema epico-omerico, ${ }^{1}$ applicandola alla scena neo-epica dei combattimenti tra Giustiniano I e gli Ostrogoti al tempo della guerra gotica (535-553).

La lettura del poema rivela un dotto lavoro di ripescaggio di moduli e topoi omerici, ma anche di rivisitazione di svariati motivi pagani e letterari, ${ }^{2}$ con conseguente adattamento alla nuova cultura di riferimento; figure del mito sono trasformate, per esempio, in creature angeliche (quali Nettunio o Saturnio...).

Tra queste, vorrei analizzare il caso della Fata Plutina, che ritengo un gioiellino di ripresa del teatro aristofaneo entro il tessuto epico rinascimentale. La storia del dio cieco della ricchezza, il protagonista dell'ultima commedia antica superstite, che, recuperando la vista, può ribaltare in positivo le sorti degli onesti, trova infatti un singolare alloggiamento tra le vicissitudini dei "cavalieri armati".

Nel libro undicesimo, nominando questa fata afflitta da cecità, una sorta di alter ego al femminile di Pluto, se ne preannuncia così una possibile guarigione:

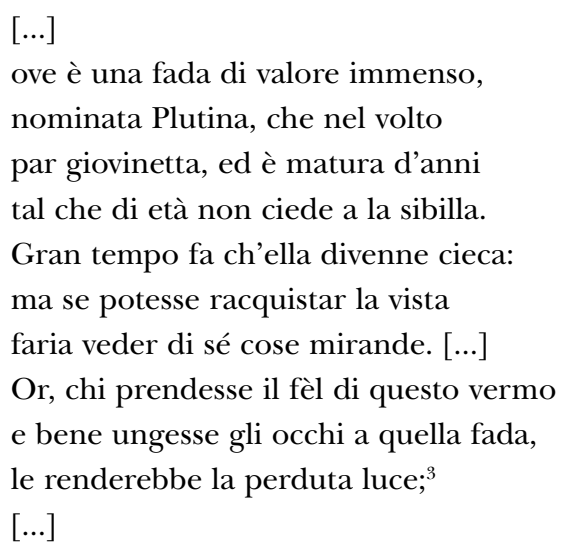

La prescrizione terapeutica mantiene il dettaglio della spalmatura dell'unguento presente nella parodia aristofanea come consueta procedura, ma all'interno di una più ampia formula, ricalcata sul genere delle "fatiche" eroiche del mito (si pensi per esempio all'im-

1 Sui riferimenti all'Iliade omerica, si veda: Baldassarri (1982); Musacchio (2003: pp. 334-352); Corrieri (2008: pp. 183-192); Pecci (2015); cfr. anche Corrieri (2008); Gigante (2010).

2 Sul senso e le fasi degli Orti Oricellari, cfr. Cambi (2015).

3 Le citazioni sono tratte da Italia liberata dai Goti, Biblioteca Italiana, online: http://ww2.bibliotecaitaliana. it/xtf/view?docId=bibit001420/bibit001420.xml [27.03.2018], che propone il testo dall'edizione veneziana del 1547 (La Italia liberata da Gotthi del Trissino, Roma, Valerio e Luigi Dorici a petizione di Antonio Macro Vincentino, maggio 1547). 
presa di Giasone e degli Argonauti per la conquista del Vello d'oro, con la lotta contro il drago e la semina dei suoi denti; qui, similmente, si parla di "bissone"):

[...] Figliuol mio, questo libretto

ha in sé descritto tutto quello incanto,

con certi versi sacri e certi modi,

che se saranno ben servati e detti

farassi andare il gran bissone a morte;

e come voi lo vederete estinto

subitamente gli trarrete il fèle

ed ungerete gli occhi a quella maga,

che le farete ritornar la vista:

[...]

Il tema ritornerà nel canto XIX:

$[\ldots]$

con la scorta del monaco arrivaro

a gli onorati alberghi di Plutina:

[...]

Ben io vorrei ch'avendo assai penato

per trar il fel del corpo a questo vermo,

che restassimo ancor quattr'altri giorni

ch'ai venticinque mancano a gir fuori,

tempo prefisso a noi da quella nimfa

per dar la morte al venenoso drago

e torli il fele e risanar Plutina.

$[\ldots]$

Nel racconto originale del Pluto si parlava di medicamento a proposito dello spergiuro Neoclide (gli veniva spalmato sulle palpebre un preparato composto di tre teste d'aglio, silfio, lentisco, aceto, vv. 716-722). Per Pluto, invece, il trattamento prevedeva l'intervento di due straordinari serpenti, in una scena carica di mistero e prodigiosa teatralità, (vv. 732-741). Il Trissino, dunque, rielabora i dati, inserendo l'elemento magico nella cornice di una ricetta medica.

Per quanto concerne l'arrivo presso Plutina, dopo la descrizione del palazzo (XI):

$[\ldots]$

d'un gran palagio, di richezza immensa:

tutte le mura eran d'argento e d'oro,

e d'oro i pavimenti e d'oro i palchi,

e di sì belle gemme eran dipinti 
che non fu visto mai cosa più ricca;

$[\ldots]$

si riporta la prima impressione conseguente alla vista di lei:

$[\ldots]$

giunsero al fine ov'era la regina,

pallida in faccia e di vedere oscuro,

con veste intorno sordide ed inculte;

$[\ldots]$

Come si può notare, se nell'originale all'uscita dal santuario di Delfi i due protagonisti della commedia greca incontravano il dio Pluto come uno straccione sporco, qui analogamente la fata, giovane nel volto, appare al primo incontro avvolta in cenci e pallida d'aspetto.

In questo senso, con interessante contrasto, la Fata sembra incarnare piuttosto l'opposta figura allegorica presente nel testo aristofaneo, la Povertà Penia, detta "livida" ('̋xpá, v. 422).

A questo punto il Trissino inserisce un passo che può ritenersi la traduzione, con qualche variante, di un dialogo del Pluto di Aristofane, in particolare i vv. 80-146, che vedremo in parallelo con il poema.

La domanda ripetuta circa l'identità del personaggio, che sorprende perché assai diverso da quanto si potrebbe presumere, trattandosi della divinità preposta al denaro:

\section{$[\ldots]$}

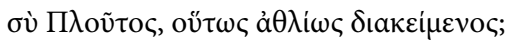

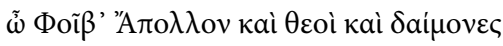

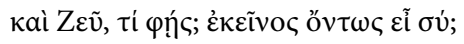

[...]

(vv. 80-82)

[Pluto, tu? Così malpreso?

O Febo Apollo, dèi e demoni,

Zeus!, che dici mai, tu quello?]

si propone anche qui:

\section{[...]}

Siete voi quell'altissima Plutina

che tanto è disiata da le genti?

E Plutina rispose: Io son pur essa.

A cui soggiunse l'onorato Achille: 
Siete Plutina voi? Si, son diss'ella.

$[\ldots]$

Poi la domanda circa l'entità del suo male e la causa:

$\mathrm{X} \rho \varepsilon \mu \dot{\lambda} о \varsigma$

$[\ldots]$

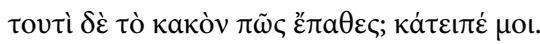

$[\ldots]$

(v. 86)

[e la tua malattia com'è andata? Raccontami!]

ritorna:

$[\ldots]$

Deh se l'eterno Dio v'adorni ed empia

gli occhi di luce acuta più che lince,

ditemi la cagion del vostro male,

che forse vi darò qualche rimedio.

$[\ldots]$

E come Pluto raccontava di essere stato accecato da Zeus perché non riconoscesse i buoni:

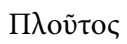

$[\ldots]$

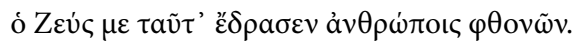

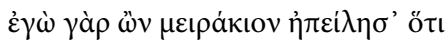

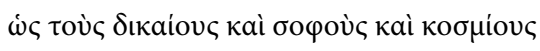

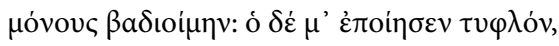

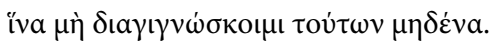

[...]

(vv. 87-91)

[Fu Zeus a farmi questo, per invidia degli uomini:

io infatti da ragazzo minacciai di andare soltanto

dai giusti e saggi e per bene; e così quello mi rese cieco,

così che non potessi distinguerli].

la fata ammette di essere stata privata della vista dal sommo Motore, perché non distinguesse gli onesti (XI): 


\section{[...]}

al tempo ch'i' era giovinetta, e vaga

di ritrovarmi dilettosi amanti,

ebbi ardir d'affirmar senza rispetto

ch'io non voleva amar se non i buoni

e i savi e i giusti, e dimorar con loro,

e fuggir tutti i perfidi e gl'ingrati;

onde 'l Motor de le superne ruote

subitamente mi privò di luce,

perch'io non conoscesse alcun di questi.

$[\ldots]$

Da notare la variazione introdotta dal poeta: trattandosi di una donna, egli ritiene preferibile parlare di amore provato per giovani onesti piuttosto che di un generico recarsi da loro.

Lo stupore per l'atteggiamento punitivo assunto dal dio riguardo alle brave persone, tanto più che sono loro a venerarlo:

\section{$[\ldots]$}

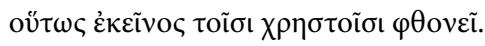

[a tal punto è invidioso dei buoni!]

$\mathrm{X} \rho \varepsilon \mu \dot{\lambda} \mathrm{\circ}$

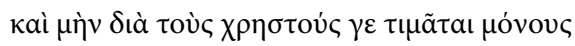

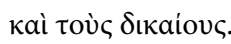

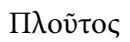

ó

(vv. 92-94)

[E dire che è dai buoni e dagli onesti soltanto

che egli riceve onori!

La penso come te...]

si riflette nei versi del Trissino (XI):

\section{[...]}

O gran disaventura de' mortali!

Pur il Signore altissimo del cielo

è solamente da le genti buone

amato ed onorato, e non da l'altre;

e poi non vuol che sian da voi vedute 
né conosciute mai, se non per caso.

$[\ldots]$

La domanda riguardo all'eventualità del recupero della vista, se cioè intenderebbe accompagnarsi, come allora, soltanto alle persone per bene (e la battuta in cui si lamenta che da molto tempo non se ne vedono):

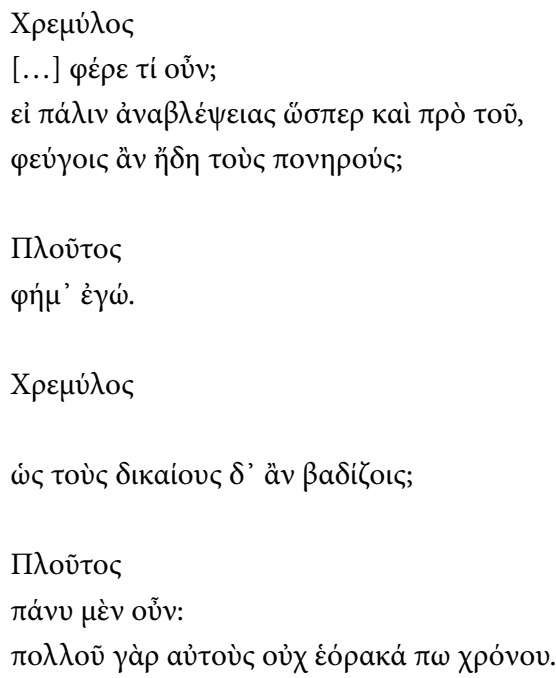

Kapíwv

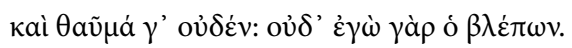

[...]

(vv. 94-99)

[ma, se tornassi a vedere come prima,

fuggiresti ancora i malvagi?

Sì!

$\mathrm{E}$ andresti dai giusti?

Certo!

È molto tempo che non ne vedo.

Niente di strano; neanch'io che pure ci vedo bene!]

si ripropone anche a proposito della Fata (XI):

$[\ldots]$

Ma ditemi, signora, s'a i vostr'occhi

si ritornasse la perduta vista,

sareste ancor di quel pensier primiero

d'amare i giusti e d'abitar con essi, 
e di fuggire i perfidi e gl'ingrati?

Si sarei, rispos'ella, che è gran tempo

che veduto non ho persona giusta.

Ed ei rispose sorridendo, e disse:

Meraviglia non è se voi che siete

priva di vista non vedete i giusti,

che noi che gli occhi avem non ne vedemo.

[...]

Parimenti, la timidezza del dio Pluto e la sua paura nei confronti di Zeus, con la risposta pronta dell'interlocutore che gli oppone lo strapotere del denaro, superiore al sommo dio:

$[\ldots]$

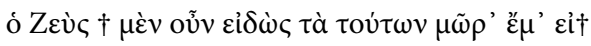

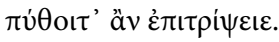

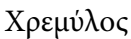

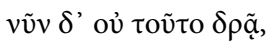

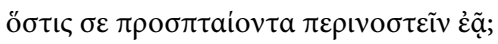

$\Pi \lambda$ กข̃นด

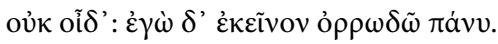

$\mathrm{X} \rho \varepsilon \mu \dot{\lambda}{ }_{\circ \varsigma}$

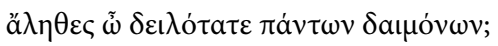

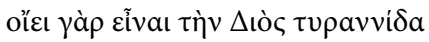

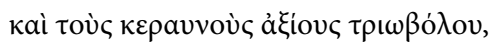

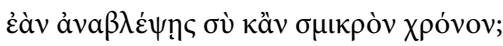

$\Pi \lambda$ oṽนด

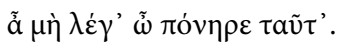

$\mathrm{X} \rho \varepsilon \mu \dot{\lambda} о \varsigma$

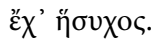

'E $\gamma \grave{\omega} \gamma$ $\alpha$ $\rho$ ảं

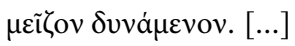

(vv. 119-129)

[Se Zeus viene a sapere queste follie mi disintegra. 
Lo sta già facendo, lasciandoti inciampare girando di qua e di là.

Non so, ma mi terrorizza.

Davvero, il più vigliacco di tutti gli dèi!

Non pensi che se tu tornassi a vedere

anche per poco tempo

il potere di Zeus e i suoi fulmini

non varrebbero un centesimo!

Canaglia, non parlare così!

Sta' calmo. Ti dimostrerò

che tu sei molto più potente di Zeus.]

risulta recuperata nel testo italiano a proposito della fata (XI):

$[\ldots]$

Ma temo, lassa, che 'l voler divino

sarà contrario molto a questa impresa;

ond'egli poi per l'arroganza vostra

potria mandarmi qualche altra ruina.

E Corsamonte: O timida che siete, voi non sapete, no, le vostre forze.

Certo, se voi racquisterete il lume, non sarà su nel ciel valor sì grande

ch'agguagliar possa la possanza vostra: [...]

Gli elenchi si moltiplicano nell'originale fino al v. 146, per poi giungere alla conclusione che tutto è nelle mani della ricchezza:

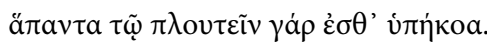

[tutto quanto dipende dalla ricchezza]

presente anche in Trissino:

$[\ldots]$

perché ogni cosa a voi s'inchina e cede.

[...] 
L'episodio della Fata del libro undicesimo (Plutina viene poi rinominata di sfuggita nel XIV libro, mentre Poro e Penia, protagonisti del mito collegati a Pluto, fanno la loro comparsa come due porte opposte nel libro XXV) mi pare, insomma, particolarmente suggestivo e interessante per comprendere la modalità duplice di reimpiego della commedia greca da parte del poeta; oltre alla conclamata originale ripresa della struttura drammaturgica ellenica sulla base di riferimento plautina della commedia Simillimi ${ }^{4}$ (naturalmente avvalendosi pure del fondamentale testo della Poetica aristotelica, da Trissino apprezzato e commentato, ${ }^{5}$ cfr. Trissino 1562), vediamo qui anche l'inserimento colto di una scena aristofanea, che non è necessariamente collegata dal punto di vista della trama con il modello, ma viene incastonata con una sua relativa autonomia grazie anche ad aggiustamenti poetico letterari.

\section{Bibliografia}

Antimaco G. (Ed.). (1864). Trissino, G. G.: La Sofonisba tragedia e i Simillimi. Milano: G. Daelli e. C. [Bologna: A. Forni 1974].

Baldassarri, G. (1982). Il sonno di Zeus. Sperimentazione narrativa del poema rinascimentale e tradizione omerica. Roma: Bulzoni.

Bertini, F. (1997). Plauto e dintorni. Roma - Bari: Laterza.

Bertini, F. (2001). Riecheggiamenti euripidei nei Simillimi del Trissino. Studi Umanistici Piceni, 21, 163-171.

Cambi, F. (2015). Gli Orti Oricellari: Un cenacolo formativo del Rinascimento. Educazione: Giornale di Pedagogia critica, 4, 7-27.

Castelvecchi, A. (1986). Trissino: Scritti linguistici (ed. A. Castelvecchi). Roma: Salerno Editrice.

Castorina, C. (2016). Sofonisba di Giovan Giorgio Trissino. Studi Giraldiani, 2, 121-152.

Corrieri, A. (2008). Rivisitazioni cavalleresche ne L'Italia liberata da' Gotthi di Giangiorgio Trissino. Schifanoia, 34-35, 183-192.

Cremante, R. (1988). Teatro del Cinquecento. La tragedia (2 Vols.). Milano - Napoli: Riccardo Ricciardi Editore.

De Amicis, V. (1897). L'imitazione latina nella commedia italiana del XVI secolo (pp. 164-166). Firenze: Sansoni.

Gigante, C. (2010). Epica e romanzo in Trissino. In C. Gigante, \& G. Palumbo (Eds.), La tradizione epica e cavalleresca in Italia (XII-XVI sec.) (pp. 291-320). Bruxelles: P.I.E. Peter Lang.

Guerrieri Crocetti, C. (1937). Giangiorgio Trissino. In Enciclopedia Treccani (Vol. 34). Roma: Treccani.

Musacchio, E. (2003). Il poema epico ad una svolta: Trissino tra modello epico e modello virgiliano. Italica, 80, 334-352.

4 Commedia che di base era modellata sui Menecmi plautini; De Amicis (1897); Bertini (1997); Bertini (2001).

5 Cfr. Trissino (1562); del Trissino si veda anche Antimaco (1864); Castelvecchi (1986); Cremante (1988). Sull'autore, Sanesi (1911); Guerrieri Crocetti (1937); Palumbo (1961); Stäuble (1986)²; Trovato (1994); Puppi (2011-2012). 
Palumbo, P. (1961). Giangiorgio Trissino. In Letteratura Italiana (Vol. 2; pp. 873-889). Milano: Marzorati Editore.

Pecci, P. (2015). Riscrittura e imitazione omerica ne L'Italia liberata dai Goti di Gian Giorgio Trissino. In Corpus Eve. Homère en Europe à la Renaissance. Traductions et réécritures [retrieved 07.04.2018 from http://journals.openedition.org/eve/1232].

Puppi, L. (2011-2012). Tiziano e Giangiorgio Trissino: un inedito ritratto per un'ipotesi. Odeo Olimpico, 28, 436-446.

Sanesi, I. (1911). La commedia (Vol. 1; p. 255). Milano: Vallardi.

Stäuble, A. (1986)². Umanistica, commedia. In V. Branca (Ed.), Dizionario critico della Letteratura Italiana (Vol. 4; pp. 344-349). Torino: Unione Tipografico-Editrice Torinese.

Trovato, P. (1994). Il primo Cinquecento. Storia della lingua italiana (ed. F. Bruni). Bologna: Il Mulino.

Trissino, G. G. (1562). La quinta e la sesta divisione della poetica del Trissino. Venezia: Ed. Andrea Arrivabene.

Ludovica Radif, Ph.D. / ludovica.radif@upol.cz

Department of Classical Philology

Palacký University in Olomouc, Philosophical Faculty

Na Hradě 5, 77180 Olomouc, Czech Republic 
